\title{
Berggebietsförderung und Ökologie - ein Widerspruch?
}

\section{Einleitung und Fragestellung}

Zentrale Grundlage der schweizerischen Berggebietsförderung ist das Bundesgesetz über Investitionshilfe für Berggebiete vom 28. Juni 1974 (IHG). Auf der Basis dieses Gesetzes werden förderungsbedürftige und entwicklungsfähige Regionen beim Ausbau ihrer Infrastruktur unterstützt. Der Bund und die Kantone - diese haben sich mit einer sogenannten Äquivalenzleistung an den Förderprojekten zu beteiligen - versprechen sich davon eine Verbesserung der Wohn- und Standortattraktivität peripherer Regionen, was letztlich die Schaffung von Arbeitsplätzen erleichtern und die Abwanderung von Arbeitskräften eindämmen soll.

Mit diesen wirtschaftlichen Stoßrichtungen steht das IHG im bekannten grundsätzlichen Konflikt mit ökologischen Anliegen. Im Vergleich zum benachbarten Ausland dürfte dieser Konflikt indessen durch klar weniger deutliche Konturen gekennzeichnet sein. Mit der Ausrichtung des sachlichen Geltungsbereiches auf die kleinräumige Infrastruktur befaßt sich das IHG nämlich in erster Linie mit relativ umweltverträglichen Investitionen. Die aus ökologischer Sicht besonders problematischen Großprojekte wie Autobahnen oder Kraftwerke sind nicht Gegenstand der Berggebietsförderung; entsprechende Investitionen laufen nicht über regionalpolitische, sondern sektoralpolitische Budgets. Hinzu kommt, daß über das IHG nicht selten Projekte abgewickelt werden, die letztlich zu einer verbesserten Umweltqualität beitragen (Kanalisationen, Kläranlagen usw.).

Der vorliegende Aufsatz soll darüber Aufschluß geben, wie die ökonomischen und ökologischen Anliegen der Entwicklungsplanung im schweizerischen Berggebiet mit Hilfe der regionalen Entwicklungskonzepte der zweiten Generation (EIDGENÖSSISCHES VOLKSWIRTSCHAFTSDEPARTEMENT 1989) noch besser unter einen Hut gebracht werden sollen. In den Kapiteln 2 und 3 werden die verschiedenen Funktionen der regionalen Entwicklungskonzepte vorgestellt. Am konkreten Beispiel der Pilotregion Thal (SO) soll dann in Kapitel 4 aufgezeigt werden, wie ökologische Fragen im Rahmen eines modernen regionalen Entwicklungskonzeptes behandelt werden können.

\section{Das regionale Entwicklungskonzept als Führungsinstrument}

In den Genuß der Investitionshilfe kommen alle diejenigen Regionen des schweizerischen Berggebietes, die über ein genehmigtes regionales Entwicklungskonzept verfügen. Das regionale Entwicklungskonzept ist das zentrale mittel- und längerfristige Führungsinstrument für die regionalen Entwicklungsträger. Es bildet die konkrete planerische und politische Grundlage für die Aktivitäten einer IHG-Region (vgl. dazu ARBEITSGRUPPE STOCKER 1973).

Als Planungsgrundlage dient das Entwicklungskonzept der Region zur Diskussion ihrer Zukunft; es soll im Sinne einer allgemeinen Marschrichtung Entwicklungsziele festlegen. Das Konzept zeigt auf, welche Entwicklungspotentiale erschlossen werden sollen und welche konkreten Maßnahmen dafür zu treffen sind. Für die Gemeinden ist das regionale Entwicklungskonzept eine Orientierungshilfe im Finanz- und Investitionsbereich. Die Privatwirtschaft kann auf der Basis klarer regionaler Entscheidungsgrundlagen sinnvolle Dispositionen für die Zukunft tätigen.

Als Koordinationsplattform fördert das regionale Entwicklungskonzept die Zusammenarbeit zwischen den Gemeinden sowie zwischen öffentlicher Hand und privaten Akteuren. Es dient als Abstimmungsinstrument entwicklungsrelevanter Massnahmen der verschiedenen staatlichen Ebenen.

Im Bereich des Vollzugs stellt das regionale Entwicklungskonzept eine wichtige Grundlage für die Gewährung der Investitionshilfe dar. Nach ihm richtet sich auch die Beurteilung von Gesuchen für Bürgschaften und Zinskostenbeiträge von Klein- und Mittelbetrieben ${ }^{1}$ und von Gesuchen um Hotel- und Kurortkredite? 2 .

Ein Blick auf die langjährige Vollzugstätigkeit der Regionen der schweizerischen Berggebiete läßt erkennen, daß sich die regionalen Entwicklungskonzepte als Führungsinstrumente grundsätzlich bewährt haben und in verschiedensten Bereichen

Christian Hanser, Dr., Brugger, Hanser und Partner, Winterthurerstraße 52, CH-8006 Zürich 
nachhaltige Entwicklungsimpulse auszulösen vermochten (Infrastrukturausbau, Industrie- und Gewerbeförderung, Landschaftsschutz usw.). In den meisten Entwicklungsregionen leisten die regionalen Entwicklungskonzepte außerdem einen nicht zu unterschätzenden Beitrag zur Verbesserung der Zusammenarbeit der Gemeinden.

Trotz grundsätzlich positiver Beurteilung sind einzelne Schwächen der regionalen Entwicklungskonzepte der ersten Generation nicht zu übersehen: In erster Linie ist auf die mangelhafte Koordination mit raumrelevanten Sachplanungen von Bund und Kanton hinzuweisen. Die sektorielle Betrachtung herrscht vor; die verschiedenen Ziele und Stoßrichtungen werden kaum auf ihre gegenseitige Verträglichkeit geprüft. Komplementaritäten und allfällige Zielkonflikte werden nicht in der notwendigen Differenzierung ausdiskutiert, was immer wieder zu Mißverständnissen führt. Als großen Mangel empfinden die mit dem Vollzug der Entwicklungskonzepte betrauten Stellen und Gremien den stark zurückschauenden Analyseteil, der eine prospektive Interpretation der regionalen Potentiale sehr erschwert. Als Schwäche erweist sich ferner der oft kaum nachvollziehbare Zusammenhang zwischen Problemen, Strategien und einzelnen Projekten ${ }^{3}$.

\section{Die Entwicklungskonzepte der zweiten Generation}

Die meisten regionalen Entwicklungskonzepte wurden in der zweiten Hälfte der siebziger Jahre erarbeitet. Inzwischen haben sich wichtige Rahmenbedingungen der regionalwirtschaftlichen Entwicklung entscheidend verändert. Vielerorts wurden in der Zwischenzeit Probleme aktuell, die in den entsprechenden Planungsgrundlagen weitgehend unberücksichtigt blieben. Mit anderen Worten: zahlreiche regionale Entwicklungskonzepte der ersten Generation sind veraltet. Vor dem Hintergrund der im vorangehenden Kapitel skizzierten Schwächen wurden in den Richtlinien des EIDGENÖSSISCHEN VOLKSWIRTSCHAFTSDEPARTEMENTES (1989) folgende Stoßrichtungen formuliert, die es bei der Überarbeitung der Entwicklungskonzepte zu beachten gilt:

- Die regionalen Entwicklungskonzepte sind in thematischer Hinsicht an die veränderte regionalwirtschaftliche Problemlage anzupassen: Das bedeutet, da $\beta$ neben wirtschaftlichen auch vermehrt ökologische und sozio-kulturelle Aspekte mitberücksichtigt werden müssen.

- Die einzelnen Konzeptbereiche sollen nicht mehr isoliert betrachtet werden. Anzustreben ist vielmehr eine vernetzte Betrachtung der verschiedenen Bereiche mit detaillierter Darstellung der gegenteiligen Abhängigkeiten.
- Zukunftsorientierte Betrachtungen erhalten ein Schwergewicht gegenüber der rückschauenden Analyse. Das Konzept trägt den überregionalen Rahmenbedingungen und den daraus abzuleitenden Einflüssen Rechnung.

Die Entwicklungskonzepte der ersten Generation wurden auf der Grundlage der von der ARBEITSGRUPPE STOCKER verfaßten Richtlinien für die Berggebietsförderung (1973) erarbeitet. Diese Richtlinien gingen in ihren Anweisungen recht weit und sahen einen strengen Planungsvorgang von Lageanalyse über Ziele und Maßnahmenkatalog bis hin zur finanziellen Machbarkeitsprüfung vor. Da mit diesem Ansatz die Individualität der einzelnen Regionen zu wenig zur Geltung kam, wurden für die Konzepte der zweiten Generation die verbindlichen Vorgaben auf ein Minimum beschränkt. Zudem soll hinsichtlich der konkreten Stoßrichtungen und Entwicklungsprojekte ein aufgaben- und problemorientiertes Vorgehen gewählt werden, das dem Handlungsspielraum der regionalen Entwicklungsträger auf realistische Weise Rechnung trägt. Die Entwicklungsplanung wird damit weniger ambitiös und bei sich ändernden Verhältnissen anpassungsfähiger. Im Hinblick auf größtmögliche Flexibilität erfolgt die Konzeptrevision in zwei Schritten: Eine erste Arbeitsphase umfaßt alle diejenigen Arbeitsschritte, die aufzeigen, wo das neue regionale Entwicklungskonzept ansetzen und Schwerpunkte bilden muß. Die Strategiediskussion ist aufgegriffen, bedarf aber noch weiterer Vertiefungen. Die zweite Phase befaßt sich dann mit den inhaltlichen Schwerpunkten der Konzeptüberarbeitung und setzt die festgestellten Befunde in ein handlungsorientiertes Konzept um.

\section{Berggebietsförderung und Ökologie: Anspruch und Wirklichkeit}

Die methodischen Vorgaben für die Überarbeitung der regionalen Entwicklungskonzepte bilden ohne Zweifel eine gute Voraussetzung für eine konsequente Berücksichtigung ökologischer Belange bei der Realisierung aller relevanten Maßnahmen und Projekte. Sowohl in der Planungsphase als auch in der Phase des Vollzugs sind die Entwicklungsträger gezwungen, ein Vorhaben unter ökonomischen und ökologischen Gesichtspunkten zu optimieren. Die nachfolgenden Ausführungen stellen am Beispiel der Pilotregion Thal dar, auf welche Weise diese Vorgaben im Rahmen eines konkreten Entwicklungskonzeptes umgesetzt werden. Eine knappe Skizze der Entwicklungspolitik in der Region Thal vermittelt die für diese Diskussion notwendige Grundlage. 


\subsection{Das EK2 der Region Thal im Überblick}

\section{Ziele}

Die entwicklungspolitischen Bestrebungen der Regionalplanungsgruppe Thal sind darauf ausgerichtet, die wirtschaftliche, politische und kulturelle Eigenständigkeit der Region auch in Zukunft zu bewahren. Man will dies erreichen, indem die vielfältigen Wohn- und Standortvorteile sinnvoll genutzt werden (vgl. dazu ausführlich REGIONALPLANUNGSGRUPPE THAL (1990, S. 12 f.).

Bereits in ihrem ersten Strategiepapier anfangs der 70er Jahre (REGIONALPLANUNGSGRUPPE THAL 1975) hat die Regionalplanungsgruppe Thal eine Reihe von Gegensätzen festgelegt, welche in ihrer Ausrichtung auch heute noch uneingeschränkt Gültigkeit besitzen:

- Im Thal soll Lebensqualität im weitesten Sinne erhalten und geschaffen werden. Den Fragen des Umweltschutzes, der Landwirtschaft sowie der Pflege der Kultur ist große Aufmerksamkeit zu schenken.

- Der Abwanderung, besonders der jungen Thalerinnen und Thaler, soll entgegengewirkt werden durch die Schaffung neuer Arbeitsplätze und Lehrstellen in der Industrie, im Gewerbe und im Dienstleistungssektor, vorab in Branchen, die heute nur schwach oder gar nicht vertreten sind.

- Die Einkommen pro Kopf der Bevölkerung sollen auf das Niveau des kantonalen Durchschnittes angehoben werden.

- Die Region Thal ist als ruhiges Wohngebiet mit vielen Entwicklungsmöglichkeiten zu fördern.

\section{Die wesentlichen Handlungsbereiche}

Ausgangspunkt der Entwicklungsanstrengungen der Regionalplanungsgruppe Thal bildet die Verbesserung der infrastrukturellen Ausstattung der Region. Auf dieser Basis wird drei, in jeder Beziehung gleichberechtigten Handlungsbereichen besondere Aufmerksamkeit geschenkt:

- Stärkung von Industrie und Gewerbe

- Förderung der Wohnbautätigkeit

- Landwirtschaft und Landschaftsschutz

Dieses Drei-Säulen-Prinzip geht davon aus, daß das Zusammenwirken aller drei Pfeiler gute Voraussetzungen für eine wohnliche und wirtschaftlich gesunde Region schafft.

Im Verlaufe der Jahre hat sich das Tätigkeitsfeld der Regionalplanungsgruppe Thal immer stärker ausgeweitet. Zu Beginn konzentrierte sich die Entwicklungspolitik auf wirtschaftliche Fragen und Projekte. Die verstärkte Auseinandersetzung mit Raumplanungs-, Energie-, Verkehrs- oder Umweltfragen, um nur einige neue Themen zu nennen, ist Ausdruck des Sachverhaltes, daß sich die Thaler Entwicklungspolitik immer mehr in Richtung eines gesamtheitlichen Ansatzes weiterentwickelte. Abb. 1 gibt einen Überblick über die im Entwicklungskonzept der zweiten Generation (EK2) behandelten Themenbereiche und die in den einzelnen Themenbereichen formulierten Stoßrichtungen.

\subsection{Der Stellenwert der Ökologie}

Dem Grundsatz der ökologischen Verträglichkeit versucht das Entwicklungskonzept der Region Thal gerecht zu werden, indem dem Themenbereich Umwelt eine Art Querschnittfunktion zugewiesen wird. Der Ökologieaspekt findet in allen Themenbereichen Berücksichtigung; jede umweltrelevante Stoßrichtung soll unter anderem auch ökologischen Anliegen Rechnung tragen. Besonders deutlich zeigt sich dieser Anspruch im Bereiche des Arbeitsmarktes. Im Hinblick auf die weitere Verbesserung der regionalen Wettbewerbsfähigkeit fordert das EK2 einerseits die Weiterführung der in der Region Thal seit Jahren erfolgreich betriebenen Industrieansiedlungspolitik.

Andererseits sind die Förderinstanzen gehalten, qualitative Ansiedlungskriterien strikte einzuhalten: Erwünscht sind ausschließlich Betriebe mit hoher Wertschöpfung, günstiger Qualifikationsstruktur, geringer Umweltbelastung und bescheidenem Flächenverbrauch (STOKAR 1990, S. 20).

In bezug auf Landwirtschaft verfolgt das EK2 der Region Thal das Ziel, leistungsfähige und naturnahe Betriebe zu erhalten bzw. zu fördern. Das Konzept geht davon aus, daß es zur Erfüllung dieser Zielsetzung notwendig ist, sowohl die wirtschaftliche Lage der Bergbetriebe zu verbessern und die allgemeinen Lebensbedingungen im Berggebiet attraktiver zu gestalten als auch standortspezifischen «naturnahen» Bewirtschaftungsweisen zum Durchbruch zu verhelfen (HANSER/MEIER 1990, S. 72 f.). Angesichts steigender Löhne und fallender Holzpreise wird es für die Thaler Forstbetriebe immer schwieriger, eine ausgeglichene Rechnung zu erzielen. Die Forstdienste der Gemeinden vermochten zwar den öffentlichen Wald bisher noch in traditioneller Weise zu bewirtschaften. In vielen Privatwäldern sind hingegen Nutzung und Pflege aus finanziellen Gründen eingestellt worden. Vor diesem Hintergrund kommt das EK2 nicht darum herum, für die Forstwirtschaft wirtschaftliche Unterstützungsmaßnahmen vorzusehen und die Erschließung der Thaler Wälder zu verbessern. Zugleich stipuliert das EK2 aber auch eine schonende und zurückhaltende Waldbewirtschaftung. Ökologisch wertvolle Waldgebiete sollen unter verstärkten Schutz gestellt werden.

Im Bereiche Verkehr sucht das EK2 einen ökonomisch und ökologisch befriedigenden Kompromi $\beta$ zwischen öffentlichem und privatem Verkehr.

In bezug auf den Tourismus strebt das EK2 eine bessere und wertschöpfungsintensivere Nutzung der 


\section{Arbeitsmarkt}

- Förderung der beruflichen Aus-, Fort- und Weiterbildung

- Schaffung besserer Voraussetzungen für die Rekrutierung von Arbeitskräften

- Verbreiterung des Angebotes an qualifizierten Arbeitsplätzen

\section{Umwelt}

Schaffung der institutionellen Voraussetzungen auf regionaler und kommunaler Ebene

- Wirksamer Vollzug von Erlassen und Empfehlungen im Bereiche des Umweltschutzes

- Gezielte Massnahmen in den einzelnen Umweltschutzbereichen

\section{Energie}

- Schaffung einer ständigen Energiekommission

- Einsetzung eines Energiebeauftragten

- Aufbau einer Energiedienstleistungsunternehmung (EDU)

- Schaffung eines Energiefonds Thal

\section{Kommunikation}

- Sensibilisierung von Wirtschaft, Nonprofit-Organisationen und Bevölkerung für neue Kommunikationstechnologien

- Förderung der Aus- und Weiterbildung in den Bereichen EDV und Telekommunikation

- Förderung von innovativen Telekommunikationsprojekten im primären, sekundären und tertiären Sektor

- Unterstützung der Bestrebungen zur Schaffung von Telearbeitszentren und Informations-Ateliers

- Unterstützung der Thaler Nonprofit-Organisationen bei der Identifikation und Realisierung von sinnvollen Telekommunikationsprojekten

Q Prüfung der Möglichkeit zur Schaffung eines regionalen Telekommunikationszentrums

- Aufbau eines "Frühwarnsystems" zur raschen Wahrnehmung neuer Chancen und Risiken
Soziales/Gesundheit

- Sicherstellung eines ausreichenden Angebotes an Plätzen in Alters und Pflegeheimen und an Alters wohnungen

- Sicherstellung aller Dienstleistungen im Spitex-Bereich auf der Basis der schon bestehenden Institutionen

- Förderung von Organisationen und Vernetzung der Sozialdienste

- Förderung der Weiterbildung

- Intensivierung der Öffentlich keitsarbeit

- Sicherstellung einer bedarfsgerechten Gesundheitsversorgung

- Bedarfsgerechter Ausbau der Hausbesuche

\section{Landwirtschaft}

- Gezielte Verbesserung der landwirtschaftlichen Produktion und der Vermarktung

a Förderung einer massgeschneiderten Bildung und Beratung für die Thaler Bergbauern

- Förderung der Erwerbskombination

- Förderung naturnaher Bewirtschaftungsweisen

- Schliessung der Lücken in der Infrastruktur und Verbesserung der Gebäudeverhältnisse

\section{Forstwirtschaft}

- Verstärkung der Pflege und Nutzung von öffentlichen und privaten Wäldern

- Förderung und Durchsetzung naturnaher Bewirtschaftungsweisen

- Verbesserung der Marktchancen für Holz

aus- und Weiterbildung

Raumplanung

- Konsequente Umsetzung Regionalplan

- Vermehrte Beachtung qualitativer Aspekte bei der Ortsplanung - Umsetzung der weiteren Planungsgrundlagen

- Aktive Bodenpolitik
Verkehr

Beschränkung der Investitionen im Bereiche Individualverkehr auf punktuelle qualitative Verbesserungen

口 Erhöhung der Wettbewerbsfähigkeit des öffentlichen Verkehrs - Bessere Verknüpfung von Individualverkehr und öffentlichem Verkehr

- Durchsetzung der Massnahmen zur Verkehrsberuhigung auf den Zufahrtsstrassen zu den Jurahöhen und Bereitstellung von Parkplätzen

\section{Kultur/Jugend/Sport}

- Förderung von Einrichtungen für ein vielfältiges kulturelles Ange bot

- Bessere Verankerung kultureller Anliegen in den Gemeinden

- Förderung der kulturellen Information und Animation

- Förderung eines jugendgerechten Umfeldes

Gehör für Jugendliche im Kreise der Repla und in den Thaler Ge meinden

- Schaffung von Jugendräumen und finanzielle Unterstützung

- Punktuelle Ergänzung der Sportanlagen

\section{Schule/Erwachsenenbildung}

- Sicherstellung und punktuelle Verbesserung des vorschulischen und schulischen Angebots

- Verbesserung der Koordination und Information im Bereiche Erwachsenenbildung

\section{Wohnungsmarkt \\ - Bodenpolitische Offensive - Sparsamer Flächenverbrauch - Fortsetzung der Wohnbauförde- rung}

Tourismus

- Erhaltung des Erholungsraumes und der natürlichen Schönheiten der Region

- Bessere Nutzung der vorhandenen touristischen Potentiale

- Verbesserung der institutionellen Rahmenbedingungen

Abb. 1 Die Themenbereiche und Stoßrichtungen des EK2 der Region Thal im Überblick. 
endogenen Potentiale an. Der Naherholungstourismus, der insbesondere an schönen Herbstwochenenden stark belastende Ausmaße erreicht, soll hingegen durch die Schaffung verkehrsfreier Räume und durch entsprechende Wegmarkierungen auf die bekannten Hauptrouten konzentriert werden. Ökologisch wertvolle Bergzonen sollen weder durch den Tourismus noch durch das Militär beeinträchtigt werden.

Diese Beispiele - sie stehen stellvertretend für alle übrigen Themenbereiche - sollen zeigen, auf welche Weise das Anliegen der ökologischen Verträglichkeit die miteinbezogenen Themenbereiche mitprägt. Im Rahmen des EK2 der Region Thal geht es nicht darum, die traditionellen entwicklungspolitischen Ansätze vor dem Hintergrund zunehmender ökologischer Probleme leicht zu modifizieren. Wo notwendig und sinnvoll, sucht das EK2 vielmehr echte strategische Neuorientierungen mit expliziter Berücksichtigung der relevanten Umweltaspekte.

Obwohl alle Themenbereiche des EK2 der Region Thal in einem pragmatischen Sinn auf Umweltverträglichkeit ausgerichtet sind, erwies es sich bei der Konzeptüberarbeitung als notwendig, einen eigenständigen Themenbereich Umwelt zu konzipieren. Der föderalistischen Arbeitsteilung zwischen Bund, Kanton und Gemeinden Rechnung tragend, ist die Strategie der Regionalplanungsgruppe Thal im Umweltbereich stark auf Koordination, Information und Beratung ausgerichtet. Sie nimmt sich organisatorischen institutionellen Fragen an. Zugleich hilft sie, Vollzugsengpässe bei der Anwendung bestehender Gesetze zu beseitigen bzw. aufzudecken. Der Entwicklungsträger übernimmt in diesem Sinne auch eine Mittlerrolle zwischen Kanton und Gemeinde.

Das EK2 setzt im Umweltbereich die folgenden drei Maßnahmenpakete ins Zentrum:

a) Schaffung der institutionellen Voraussetzungen für eine wirksame Umweltpolitik auf regionaler und kommunaler Ebene

Operative Aufgaben im Zusammenhang mit dem Umweltschutz auf regionaler Ebene werden durch das Regionalsekretariat ausgeführt. Damit die daraus sich ergebenden Arbeiten kompetent und zeitgerecht erledigt werden können, ist dem Sekretariat eine Umweltberatungsstelle anzugliedern. Die Tätigkeit der Umweltberatungsstelle erstreckt sich auf die aktive Wahrnehmung von Aufgaben in den Bereichen Koordination, Beobachtung, Information, Beratung sowie Aus- und Weiterbildung. Sie ist primär als Anlaufstelle für die Mitglieder der Gemeindeexekutiven und der kommunalen Umweltkommissionen oder -beauftragten gedacht. Die unmittelbare Beratung von Bevölkerung und Wirtschaft steht nicht im Zentrum.
Die Gemeinden sind aufgerufen, entweder die Schaffung einer Umweltschutzkommission oder die Bestimmung eines Umweltbeauftragten in die Wege zu leiten. Der bereits bestehende, bis anhin informelle Kreis der Umweltkommissionspräsidenten und -beauftragten ist als regionale "Arbeitsgruppe für Umweltfragen» zu institutionalisieren. Für kommunale Fachkommissionen (Baukommissionen, Umweltschutzkommissionen, Naturschutzaufseher usw.) sind Einführungs- und Weiterbildungsangebote bereitzustellen. Die einzelnen Umweltschutzbeauftragten nutzen die regionale Arbeitsgruppe Umwelt als Forum für den Erfahrungsaustausch.

\section{b) Wirksamer Vollzug von Erlassen und Empfehlungen im Bereiche des Umweltschutzes}

Der Kanton Solothurn und die Gemeinden der Region Thal ergreifen vermehrt die Initiative zu einem nachhaltigen Schutz der bestehenden Naturreservate. Im Hinblick auf die Schaffung wirksamer Pufferzonen und die Vernetzung der verschiedenen Reservate wird man nicht darum herumkommen, zusätzliche Flächen für Naturschutzzwecke auszuscheiden. Der Regionalplanungsgruppe Thal kommt dabei aufgrund des Regionalplans ein Beratungs- und Koordinationsauftrag zu.

\section{c) Gezielte Maßnahmen in den einzelnen Umweltschutzbereichen}

- Luftreinhaltung: Die Luftreinhaltung ist eine kantonale Aufgabe. Die Gemeinden sind subsidiär tätig. Ihre Zuständigkeit umfaßt die Feuerungskontrolle und die Vollzugshilfe im Vorsorgebereich (z. B. Energiesparen). Das Schwergewicht der Tätigkeit der Region liegt bei Information und Koordination sowie unterstützenden Maßnahmen (Energie, Verkehr).

- Lärm/Erschütterungen: Für den Lärmschutz sind in erster Linie die Kantone zuständig. Die Gemeinden haben u. a. im Rahmen des Baubewilligungsverfahrens den Emissions- und Immissionsschutz durchzusetzen. Die Region wird vermehrt in Fragen des militärischen Flug- und Schießlärms aktiv werden sowie Gespräche mit Schießplatzwarten über Vollzugsprobleme bei der Kontrolle militärischer Aktivitäten organisieren.

- Boden: Der Bodenschutz ist Sache der Kantone. Für die Gemeinden bestehen infolge der Komplexität der Fragen kaum Arbeitsbereiche. Auf regionaler Ebene können z. B. Aufgaben wie das Erstellen einer Bodenschutzdokumentation, die Organisation von Weiterbildungsveranstaltungen für Landwirte oder die Beratung der Umweltschutzkommissionen in Fragen der Bodenerrosion, der Bodenbelastbarkeit, Altlastenproblematik usw. wahrgenommen werden. 
- Wasser: Der Schutz der Gewässer fällt in die Zuständigkeit der Kantone. Gemeinden sind für die Frischwasserversorgung sowie für Abwasserentsorgung und -reinigung zuständig. Der Handlungsspielraum für die Region und die daraus sich ergebenden Aufgaben sind beträchtlich: Renaturierung und Revitalisierung von Bächen, Beratung der Wasserkommission, Erarbeitung von Richtlinien für den Klärschlammaustrag, Konzept über die Abwasserbehandlung usw.

- Landschaft/Ökologisches Potential: Der Schutz der natürlichen Umwelt obliegt primär den Gemeinden und der Region. Der Kanton ist u. a. verantwortlich für die Raumplanung und legt die wichtigsten Schutzobjekte fest. Die Region hat sich weiterhin für den Vollzug des Regionalplans einzusetzen und die Erarbeitung der kommunalen Naturinventare zu koordinieren.

- Abfälle: Abfallbewirtschaftung ist nach geltendem Recht Sache der Gemeinden; der Kanton ergreift die notwendigen Maßnahmen zur Sondermüllentsorgung. Das Sekretariat der Regionalplanungsgruppe koordiniert die Abfallbewirtschaftung im Thal; es wird versucht, eine einheitliche, ökologisch sinnvolle Entsorgung einzuführen und langfristig ${ }^{4}$ sicherzustellen.

- Umweltgefährdende Stoffe/Gifte: Die Kantone sind verantwortlich für den Vollzug des eidgenössischen Giftgesetzes und die sogenannte Stoffverordnung. Den Gemeinden fällt ein Informationsauftrag zu. Seitens der Region sind z. B. regelmäßig Sonderabfall-Sammelaktionen durchzuführen.

- Umweltrisiken/Chemierisiken: Der Kanton hat ein Inventar zu erstellen, in welchem die diesbezüglichen Risiken dargestellt sind. Mit den betreffenden Firmen, Institutionen usw. ist eine wirksame Katastrophenvorsorge aufzubauen. Gemeinden haben sich regional koordiniert mit Chemiewehr und Gasschutz auszustatten.

\section{Schlußfolgerungen}

Die dargestellten Beispiele verdeutlichen, daß die regionalen Entwicklungskonzepte der zweiten Generation durchaus als geeignete Grundlage für eine umweltverträgliche Berggebietsförderung anzusehen sind. Zumindest auf methodischer Ebene stimmen Anspruch und Wirklichkeit überein.

Auch auf der Ebene des praktischen Vollzugs können die regionalen Entwicklungsträger einen wert- vollen Beitrag zur Wahrung bzw. Stärkung ökologischer Ressourcen leisten. Dabei geht selbstverständlich jede Region anders vor. Sie wird um so mehr «grüne Ziele» stark gewichten, je eher sie im wirtschaftlichen Bereich erfolgreich ist und je eher die existenziellen wirtschaftlichen und infrastrukturellen Probleme überwunden sind.

Bei aller positiven Würdigung darf man indessen nicht übersehen, daß den regionalen Entwicklungsträgern die Instrumente fehlen, um die großen Umweltprobleme unserer Zeit einer Lösung näher zu bringen. Maßnahmen auf regionaler Ebene können nationale oder sogar übernationale Eingriffe zugunsten der Umwelt ergänzen, nicht aber ersetzen.

\section{Anmerkungen}

1 Bundesgesetz über die Bürgschaftsgewährung in Berggebieten vom 25. Juni 1976 (div. Änderungen)

2 Bundesgesetz über die Förderung des Hotel- und Kurortkredites vom 1. Juli 1966 (div. Änderungen)

3 Ein zusammenfassender Überblick über Stärken und Schwächen der regionalen Entwicklungskonzepte der ersten Generation liegt vor bei HANSER (1988, S. 10 f.)

${ }^{4}$ Ein entsprechendes Abfallkonzept liegt inzwischen bereits vor (vgl. STOKAR 1990).

\section{Literatur}

ARBEITSGRUPPE STOCKER (1973): Leitlinien für die Berggebietsförderung, Bern.

EIDGENÖSSISCHES VOLKSWIRTSCHAFTSDEPARTEMENT (1989): Richtlinien für die Berggebietsförderung, Bern.

HANSER, C. (1988): Entwicklungspolitik in der Region Thal (SO). Ein Überblick über Erfolge, Mißerfolge und Probleme. In: DISP Nr. 94

HANSER, C./MEIER, R. (1990): Grundlagenbericht zum Schwerpunktbereich Landwirtschaft im Rahmen des EK2 der Region Thal (SO), Balsthal/Zürich.

REGIONALPLANUNGSGRUPPE THAL (1990): EK2-Anhaltspunkte, Balsthal.

REGIONALPLANUNGSGRUPPE THAL (1990): EK2 Schlußbericht, Balsthal.

REGIONALPLANUNGSGRUPPE THAL (1975): Thal 2000, Balsthal/Brugg.

STOKAR, M. (1990): Das solothurnische Thal - Beispiel einer aktiven Förderpolitik. In: Schweizer Journal, Mai.

STOKAR, M. (1990): Regionales Abfallkonzept Thal, Balsthal. 